\title{
CORRESPONDENCE
}

\section{THE GAS TURBINE PAPERS AND DISCUSSION}

To The Editor:-

14 th April, 1946.

No one has reason to complain of Dr. Hooker's enthusiasm for the simple jet engine as long as it does not lead to a false supposition that the problems of its application to civil aircraft have already been solved.

Without doubt the time is ripe for building experimental civil aircraft; for example, a high speed mailplane with jet engines. One hopes that several projects of this kind will be sponsored in the near future because it is clearly the only way to assess and develop the safety, economy and reliability of the engine and its appropriate aircraft.

It appears as though the pure jet and the propeller turbine will require a period of some years before we see them operating as regularly and universally as the piston engine is to-day, but their popularity will grow as their problems are solved. These problems fall roughly under four headings.

1. Economy of first cost and maintenance have fallen, short of expectations. A good deal of development and rationalisation is required by designers and metallurgists to bring down the cost of blades, discs and combustion chambers. The engine auxiliaries are, as yet, a bit reminiscent of piston engine components. The design of starting systems and fuel systems and controls does not compare yet with the gas turbine itself in regard to basic simplicity, lightness and compactness. In developing the design of engine and auxiliaries alike, attention must be devoted to the right balance between lightness and good serviceability.

2. Tha low idling torque and the inertia of the rotors of turbine engines and their different handling characteristics, at first, will cause a certain amount of apprehension to all but a few highly trained military and test pilots. The method of control used on piston engines does not necessarily suggest the best solution for the gas turbine and our present technique of handling engines in the air will most likely need to be revised.

3. Operators are being pressed to contend with a large number of new conditions at one and the same time and, so far, without much evidence upon which to judge their effects. Naturally they wonder how far the experience gained with the gas turbine under service conditions is going to apply to civil use. We are setting out ta achieve overhaul periods similar to those of the best reciprocating engines, but the operator has not yet been convinced of the certainty or consistency of the overhaul period and has little information upon which to base the size and cost of terminal and intermediate service stations, or the nature and extent of spares and equipment.

4. It appears as though the gas turbine engine driving a propeller will provide the motive power for short ranges where the distance involved and the weight of a pressure cabin argue against flying at high altitudes. But in order to take full advantage of the pure jet engine for medium and long ranges, it will be necessary to develop the high altitude, air-conditioned cabin. There is a danger of under-estimating the difficulty of constructing such a cabin which would be acceptable for general use and the choice still lies open at this date between the pure jet and some form of propeller turbine with a heat exchanger or perhaps the compound engine. Both of these would permit long distances to be flown at lower altitudes where the pressure cabin problem would be less acute. This is the justification for proceeding with the development of heat exchanzers and with an engine such as has been 480 
proposed by Dr. Ricardo. One feels, however, that the ultimate answer will not be altogether fairly judged on economic factors alone and after all the factual costs have been assessed we shall be prepared to pay a little more for smoother, quieter and quicker travel and for the mere satisfaction of being rid of the propeller.

D. R. Amor, Associate Fellow (Roy Fedden, Ltd.)

To The Editor:-

7 th May, 1946.

After reading the most interesting proposals put forward by Mr. Ricardo in his recent paper on "Turbine Compounding of the Piston Aero Engine" one is left with the feeling that the admirable brevity and conciseness with which they have been presented might make the project too attractive to minds less familiar with thermodynamics than the author's.

At first sight it would appear that two blacks are being made into a white, for Mr. Ricardo has purported to show how, by compounding with an inefficient and uneconomical turbine engine an inefficient and uneconomical two-stroke compression-ignition engine, a power unit of transcendental overall economy can be obtained.

Various practical difficulties which militate against this, however, are likely to bring it down to more mundane levels.

In the first case, it is not so generally appreciated as might be supposed that the turbine engine is internally cooled, whereas the piston engine, neglecting for the moment those types which make abundant use of the lubricating oil, is externally cooled. In this respect the airscrew can be compared with the compressor of the turbine engine in that part of its function is to deliver air for cooling the engine. Granted that the two-stroke engine will receive a large measure of internal cooling from the excess air which is supplied, nevertheless, the problem of cooling the cylinder of a Mercury-sized engine producing 4,000 h.p. will be one of considerable difficulty.

Moreover, in the estimate of overall weight it is important to take full account of the inter-cylinder baffling and exhaust arrangments necessary to achieve satisfactory cooling. The weight of cowling, baffling and exhaust system for an air-cooled engine is of the order of 17 per cent. to 20 per cent. of the dry weight of the engine, so that an appreciable amendment needs to be applied to the weight estimates.

Incidentally, when comparisons are made of the fuel economy and weight of piston and turbine engines, the piston engine should be debited with its cooling drag horse power and the weight of these items, the equivalents of which are already built integrally into the turbine engine.

One last point-advantage has already been taken by the R.A.F. of the reduced ground maintenance staff needed for turbine engines. The re-introduction of a piston engine, simple though it may be, but penalised by the need for more involved exhaust gas piping (never a joy), and requiring probably more complicated gearing, would inevitably require larger ground crews than ever before.

While compounding will always have its attractions and its adherents, success in the aviation field has so often come to the man who has limited his objective and who has concentrated his efforts on a narrow front, that there are strong grounds for assuming that the best results will be achieved by more orthodox power units of greater simplicity, and very probably of the same ultimate overall efficiency as the compounded unit.

Frank Nixon, B.Sc., M.S.A.E., Fellow (Rolls-Royce, Ltd.) 